\title{
SPHERICAL ROLLING ROBOTS: DIFFERENT DESIGNS AND CONTROL ALGORITHMS
}

\author{
Y. KARAVAEV, I. MAMAEV \\ Kalashnikov Izhevsk State Technical University, Izhevsk, ul.Studencheskaya 7, 426069, Russia \\ E-mail: karavaev_yury@istu.ru,mamaev@rcd.ru
}

A. KILIN, E. PIVOVAROVA

Udmurt State University, Izhevsk, ul. Universitetskaya 1, 426034, Russia

E-mail: aka@rcd.ru, l.n.pivovarova@gmail.com

\begin{abstract}
This paper presents a review of papers devoted to the creation and investigation of spherical robots. Owing to their structural features, namely, geometric symmetry and resistance of actuating mechanisms and elements of the control system against aggressive environmental conditions, robotic systems of this type have a high potential of being used in problems of monitoring, reconnaissance, and transportation on Earth and other planets. A detailed description is given of the structures of prototypes of spherical robots which use different actuation principles: a spherical robot with an internal pendulum mechanism, a spherical robot with an internal omniwheel platform, a spherical robot with internal rotors, and a spherical robot of combined type. Experimental results are presented to give an estimate of the possibility and efficiency of controlled motion. The applied use of spherical robots depending on the type of the actuating mechanism is discussed.
\end{abstract}

\section{Introduction}

Recently there have been a large number of publications devoted to investigating and developing mobile spherical robots. Interest in spherical robots stems from their structural features, which considerably expand the scope of their application using fairly simple design concepts. The full geometric symmetry of the spherical robot makes it possible to implement omnidirectional motion, which for some modifications can be performed without additional expenditures of energy.

Another, no less important, feature is that all mechanisms of spherical robots and all elements of the control system are located inside a hermetic spherical shell, which ensures their absolute protection against aggressive environmental conditions (humidity, dust, temperature). This in turn determines the spheres of application of such mobile robots: robots for reconnaissance, monitoring and delivery under conditions of Earth and other planets, mobile transportation systems to be used under constrained conditions limiting their maneuverability, etc.

In recent decades, efforts have been made to develop various types of actuating mechanisms and control algorithms to set spherical robots in motion. For a detailed review of relevant literature, see [1-5].

All well-known actuating mechanisms of spherical robots can be divided into three groups:

- control by moving the center of mass,

- control by generating variable gyrostatic momentum,

- control by deforming the shape of the ball. 
Robots using the first control scheme are in most common use. To move the center of mass inside the sphere, one uses pendulum-type systems, sliders, skids on which the mass moves, and various vehicles rolling inside the ball [3-14], some uses fluid [16]. For example, the first spherical robot that is controlled by moving the center of mass was developed in 1996 by the authors of [9]. The robot had one active wheel and one swivel wheel. The active wheel had a point of contact with the sphere in the lower part with a control module above it, and the swivel wheel was in contact with the sphere in the upper part. Over the years this principle of controlling spherical robots has gained wide-spread acceptance, many robots with various internal wheel platforms have been designed, and control schemes and algorithms have been improved. Ample use is made of spherical robots -- Sphero toys with an internal two-wheeled platform, which can be remotely controlled from a smartphone [17].

Robots falling in the second category move by generating variable gyrostatic momentum [17-22], which is ensured by internal rotors (gyrostats). There has been a lot of research on this principle (in particular, the algebraic controllability of this system has been shown and control laws [17-19] have been obtained), but in practice such robots possess low efficiency and are of interest mainly from the point of view of the development of nonholonomic mechanics.

The third control scheme that has become wide-spread recently is realized by deforming the shape of the ball [23]. Owing to a soft shell, the spherical robot can move and even jump by deforming it. This group also includes spherical robots with variable shell shape, in particular, a spider robot whose structural design makes two modes of motion possible: walking and rolling [24]. To move in the rolling mode, the robot must arrange its extremities, which on the outside are segments of a sphere, in such a way that a sphere is formed, and must maintain rolling by alternately pushing off from the surface.

Despite the simplicity of some designs of spherical robots, the control of such devices is impossible without understanding the dynamics of their motion. This is confirmed by the results of the few experimental investigations with prototypes of spherical robots, in which the error of positioning reached $50 \%$ when control was performed using only kinematic models. In this paper we consider four modifications of spherical robots, which were investigated in the Laboratory of Nonlinear Analysis and Designing of New Types of Vehicles in Izhevsk. Analysis of the developed dynamical motion models has made it possible to develop control algorithms for each spherical robot, which were later verified in practice using purpose-built full-scale specimens. This made it possible to estimate the capabilities of each modification and to determine the technical data and requirements for the control algorithms and control systems.

\section{Results}

2.1. A spherical robot with a pendulum propulsion device

The principle of actuating the spherical robot by means of internal pendulum mechanisms involves a large number of modifications [3-14]. In the above-mentioned papers, one can find extensive references to the relevant literature, an overview of existing modifications, and a description of their special features and shortcomings.

A new model of the spherical robot with a pendulum mechanism and with three degrees of mobility has been built in the Laboratory of Nonlinear Analysis and Designing of New Types of Vehicles. A photograph of the full-scale specimen and a three-dimensional model of the internal mechanism are presented in Fig. 1. Figure 2 shows the structural design of the actuating mechanisms that enable a controlled variation of the position of the pendulum. 



Figure 1. A spherical robot with a pendulum propulsion device: a) 3D model, b) photograph of a prototype

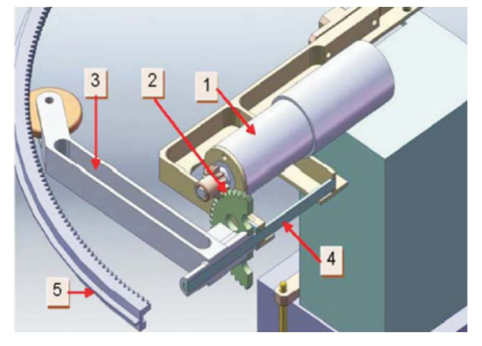

a)

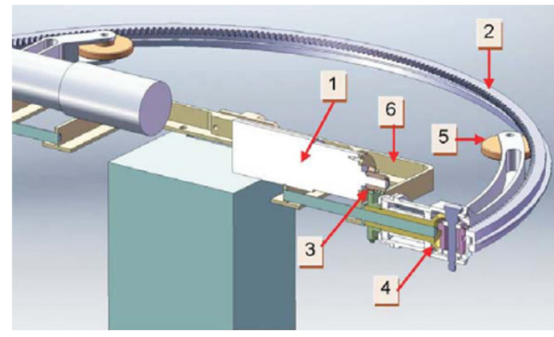

b)

Figure 2. The structural design of the actuating mechanisms of the spherical robot with a pendulum propulsion device: a) actuator for variation of latitude, b) actuator for variation of longitude. Figure 2a: 1 gearmotor (72:1), 2 - cylindrical gear drive (4:1), 3 - rocker, 4 - axle, 5 - gear with an internal gearing; $2 \mathrm{~b}$ : 1 gearmotor $(4,4: 1), 2$ - gear with an internal gearing; 3 - cylindrical gear drive $(2,3: 1), 4$ - conical gear drive, 5 rollers, 6 - frame.

The power source and all elements of the control system are located in the moving part of the pendulum. This forms an out-of-balance, which causes the spherical shell to move.

Theoretical investigations were carried out for a more complicated model of the spherical robot with a Lagrange pendulum placed inside. A dynamical model featuring the motion of this modification of the spherical robot is presented in [11,14], where an analysis of the stability and controllability of the spherical robot is carried out. Using the results obtained, basic maneuvers (gaits) have been developed to implement motion along a predetermined trajectory. Each gait enables motion along the terminal segment of the trajectory of the robot within one oscillation of the pendulum and is given by the angle of its deviation in the vertical and horizontal planes. By combining gaits one can ensure motion along a predetermined trajectory.

\subsection{A spherical robot with internal rotors}

The spherical robot is a hollow spherical shell inside which three rotors with mutually perpendicular axes of rotation are placed in the equatorial plane. A three-dimensional model and a photograph of a full-scale specimen are presented in Fig. 3. Each rotor is set in motion by a DC motor with a reduction gear. The structural design of the system under consideration satisfies a number of restrictions:

- the center of mass of the sphere + rotor system lies at the geometric center of the sphere;

- all rotors are identical and axisymmetric, and the axes of rotation coincide with the axes of symmetry, i.e., rotation does not change the mass distribution of the system;

- the axes of rotation of the rotors are noncoplanar, and the angular velocities act as controls. 


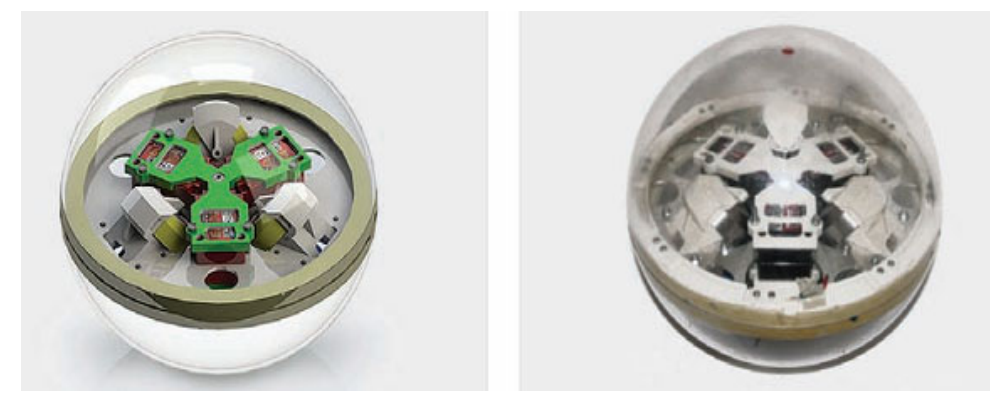

Figure 3. Design of a spherical robot with internal rotors

The design of the spherical robot is simple, and the only difficulty is to ensure absolute balance, i.e., to place the center of mass of the system at the geometric center of the spherical shell.

A detailed derivation of equations of motion of this modification of the spherical robot can be found in [17] (for the case of rolling without slipping (nonholonomic model)) and [18] in the presence of friction forces. The equations of motion are written in the form of Ferrers equations in quasi-velocities with undetermined multipliers. In addition, these papers present estimates of the stability and controllability of a spherical robot with three rotors, show the analytical dependence of control actions (angular velocities or angular momenta of the rotors) for the implementation of motion along the trajectory that is a function of time. The rolling of the spherical robot is demonstrated in Movie https://youtu.be/WdoOOK6CsLY.

\subsection{A spherical robot with an internal omniwheel platform}

A distinctive feature of the spherical robot with an internal omniwheel platform is its enhanced maneuverability, i.e., the possibility of motion of the spherical robot in any direction without turns. This advantage could be achieved by placing a vehicle with special omniwheels (also referred to as Mecanum wheels) inside a hollow spherical shell.

The motion of mobile robots with omniwheels on a plane is well studied. However, to ensure a constant contact of rollers of such a wheel with the inner surface of the spherical shell, the shape of the roller has been changed in comparison with the wheel for motion on a plane. A three-dimensional model of the spherical robot with an internal omniwheel platform and a photograph of a full-scale specimen are presented in Fig. 4.

For the control of such spherical robots, kinematic equations of motion [4] are used. However, control algorithms constructed on the basis of kinematic models without technically complicated feedbacks are in practice of little use due to oscillation of the spherical robot in the process of motion.

The dynamical model of a spherical robot with an internal omniwheel platform as a system consisting of many bodies is complicated. A special feature of the control algorithm of the spherical robot using the dynamical model is that after termination of the rolling motion along the predetermined trajectory and after deactivation of control the spherical robot can continue free motion, which is generally chaotic [4]. The algorithm of controlling the spherical robot using gaits (basic maneuvers) has made it possible to eliminate this drawback. This method consists in calculating control actions that cause the spherical robot to move according to a stationary solution (in a particular case, to stand) at the initial and final instant of time. Results of testing of the control algorithm using gaits are presented in [27]. The motion of the spherical robot with an internal omniwheel platform is demonstrated in Movie https://youtu.be/LrwKUXOtIKU. 

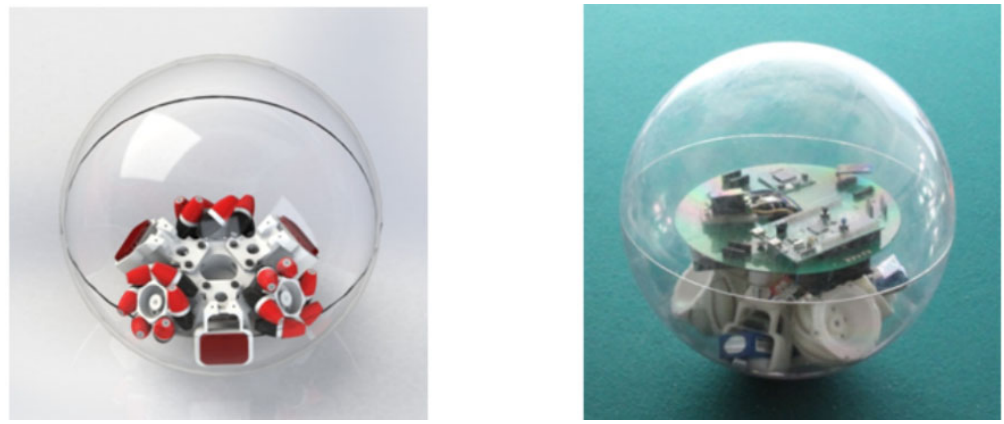

Figure 4. Design of a spherical robot with an internal omniwheel platform

\subsection{A spherical robot with a combined propulsion device}

The structural design of a spherical robot with a combined propulsion device is shown in Fig.5. The motion of the spherical robot is achieved by means of an internal two-wheeled vehicle moving in the equatorial plane along a purpose-built rim. The cross-section of the rim and the material of which it is made ensure the absence of slipping of the wheel even if the spherical robot encounters an obstacle.
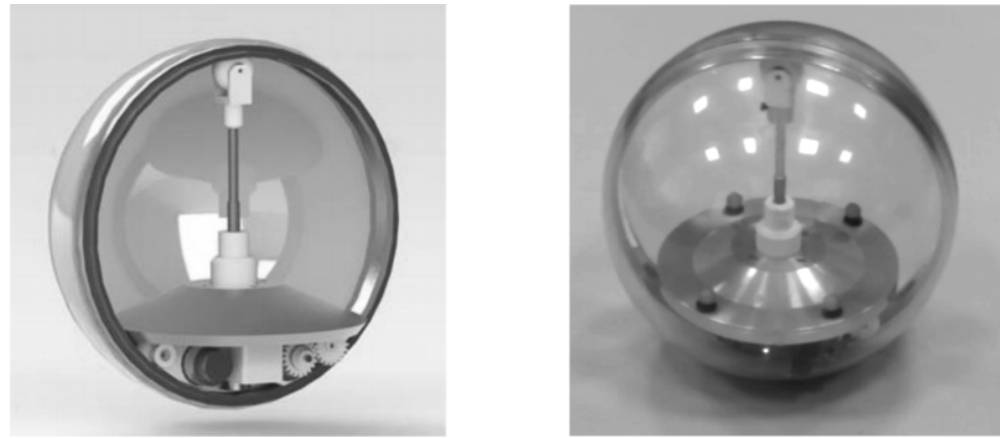

Figure 5. Design of a spherical robot of combined type

Turns of the spherical robot are made possible by generating an inertial propulsive force (torque) in ensuring an accelerated motion of the flywheel installed on the vehicle.

The control algorithm of the combined spherical robot was developed using the dynamical model of a spherical robot with a Lagrange pendulum, which was obtained for the spherical robot of pendulum type.

Results of theoretical and experimental investigations of the spherical robot with a combined propulsion device are presented in $[11,14,31]$. An example of the motion of the spherical robot of combined type along a predetermined trajectory is shown in the video file Movie https://youtu.be/3uTNqKGGwmo.

\section{Discussion}

The research results have confirmed the adequacy of the developed control algorithms on the basis of dynamical nonholonomic models of motion. The error of positioning for the above-mentioned modifications of spherical robots did not exceed $10 \%$, and can be minimized in the future by introducing velocity and position feedbacks into the control system. However, it should be noted that each modification has its drawbacks.

A significant drawback of the spherical robot of pendulum type is that it is difficult to switch over to a different mode of motion at an arbitrary instant of time (for example, if the velocity has reached a prescribed value), since it may happen that the pendulum is not in 
the lower position. In addition, the maneuverability of this type of the spherical robot can be ensured only if small-size and high-torque electric drives are used, which is technically not always possible. The structure of this spherical robot is the most complicated as compared to the other modifications considered in this paper. A large number of power transmissions and structural components requires wear resistance, rigidity and accuracy of manufacturing.

Despite the results of theoretical analysis of the dynamics of the spherical robot with internal rotors and despite successful experiments with full-scale specimens, there are a number of technical problems, which can be a significant obstacle to creating spherical robots with the main propulsion device in the form of several noncoplanar rotors:

- the necessity of fine balancing of the spherical robot and of determining its tensor of inertia;

- the difficulty of determining the coefficients of friction, since in the general case the robot performs rolling motion while rotating relative to the vertical axis (spinning); moreover, slipping can arise;

- fast and well controlled motion of the spherical robot can only be achieved if the moment of inertia of each rotor constitutes a major part of the total moment of inertia of the spherical robot, which is technically difficult in the case of three rotors.

The spherical robot of combined type and the spherical robot with an internal omniwheel platform are the most promising from the point of view of practical application. The latter possesses enhanced maneuverability, but has a high price due to the difficulty of manufacturing rollers with a special shape depending on the inner radius of the shell.

The main reason for the discrepancy between the results of theoretical simulations and experimental results is the necessity of taking into account the friction forces acting on the spherical robot. This conclusion is also relevant to all above-mentioned modifications of spherical robots, since the friction force arising during the motion of a spherical body on the surface is generally complex in nature. In the process of motion of such bodies on a horizontal surface, transitions between the mode of rolling without slipping and spinning to rolling with spinning or to slipping are possible. An important factor that has a significant, but not always predictable, influence on the motion pattern is the ratio of massgeometric parameters of the system. Specifically, for systems that are simpler than a spherical robot with internal actuating mechanisms, such phenomena as turn, overturn, loss of contact with the surface etc. have been detected. Detailed research results on such systems are presented in [32-34].

\section{Materials and Methods}

The main technical data of the developed spherical robots are presented in Table 1. To estimate the efficiency of the propulsion devices of spherical robots, the mechanisms were placed in shells of the same diameter $-300 \mathrm{~mm}$. The shell was manufactured of transparent PET material, since markers of the optical motion capture system were installed on the frames of the mechanisms inside the shell. The shell was $4 \mathrm{~mm}$ thick. The technical data of the spherical robots and their components are presented in Table 1. Control commands in the form of angular velocities of rotation of the drives were transmitted in the form of precalculated time-discrete values via a wireless communication channel from a control computer.

The motion capture system made it possible to obtain the trajectory and the orientation of the spherical robots in the process of motion and thus allowed us to estimate the control algorithms developed on the basis of the theoretical models. 
Table 1. Comparison of the technical data of mobile robots developed in the Laboratory of Nonlinear Analysis and Designing of New Types of Vehicles.

\begin{tabular}{|c|c|c|c|c|}
\hline Design & $\begin{array}{l}\text { Spherical robot } \\
\text { with an internal } \\
\text { pendulum }\end{array}$ & $\begin{array}{l}\text { Spherical robot with } \\
\text { internal rotors }\end{array}$ & $\begin{array}{l}\text { Spherical robot with } \\
\text { an internal omniwheel } \\
\text { platform }\end{array}$ & $\begin{array}{l}\text { Spherical robot of } \\
\text { combined type }\end{array}$ \\
\hline $\begin{array}{l}\text { Diameter of the shell, } \\
\mathrm{mm}\end{array}$ & 300 & 300 & 300 & 300 \\
\hline Mass, $\mathrm{kg}$ & 6.5 & 4 & 4.2 & 5.4 \\
\hline Maximal velocity, $\mathrm{m} / \mathrm{s}$ & 0.5 & 0.3 & 0.6 & 0.5 \\
\hline Power sources & $12 \mathrm{~V}, 5 \mathrm{~A}$ & $11.1 \mathrm{~V}, 15 \mathrm{~A}$ & $33.3 \mathrm{~V}, 5 \mathrm{~A}$ & $11.1 \mathrm{~V}, 10 \mathrm{~A}$ \\
\hline Time of autonomy, $\mathrm{h}$ & 4 & 1 & 6 & 4 \\
\hline Master controller & PIC32MX120 & STM32F100 & STM32F103 & LPC 1768 \\
\hline $\begin{array}{l}\text { Communication } \\
\text { channel }\end{array}$ & WiFi & Bluetooth & Bluetooth & Bluetooth \\
\hline Type of motor & $\begin{array}{l}\text { DC motor with a } \\
\text { reduction gear } \\
(4.4: 1),(72: 1)\end{array}$ & $\begin{array}{l}\text { DC motor with a } \\
\text { reduction gear } \\
(10: 1)\end{array}$ & Step motor & $\begin{array}{c}\text { DC motor with a } \\
\text { reduction gear } \\
(34: 1)\end{array}$ \\
\hline Number of motors & 3 & 3 & 3 & 2 \\
\hline
\end{tabular}

\section{Acknowledgments}

The work of Yu.L. Karavaev (Section 2) was funded by the Russian Science Foundation under grant 18-71-00096, the work of I.S. Mamaev is supported by the RFBR under grant 18-0800999-a the work of A. A. Kilin and E. N. Pivovarova was carried out within the framework of the state assignment of the Ministry of Education and Science of Russia (FEWS-2020-0009).

\section{References}

1. Chase R., Pandya A. "A review of active mechanical driving principles of spherical robots", Robotics, 2012, vol.1, no.1, pp.3-23.

2. Crossley V.A. "A literature review on the design of spherical rolling robots". Pittsburgh, Pa., 2006, 6pp.

3. Ylikorpi T., Suomela J. "Ball-shaped robots. Climbing and walking robots: Towards new applications", H.Zhang (Ed.). Vienna: InTech, 2007. P.235-256.

4. Karavaev Yu.L., Kilin A.A., "The dynamics and control of a spherical robot with an internal omniwheel platform”, Regul. Chaotic Dyn., 2015, vol.20, no.2, pp.134-152.

5. Ylikorpi T., "Mobility and Motion Modelling of Pendulum-Driven Ball Decoupled Models Robots: for Steering and Obstacle Crossing", Doctoral Dissertations, School of Electrical Engineering, 2017, 251 p. ISBN 978-952-60-7616-4

6. Zhan Q. "Motion planning of a spherical mobile robot. Motion and operation planning of robotic systems", G.Carbone, F.Gomez-Bravo (Eds.). Cham: Springer, 2015, P.361-381.

7. Bicchi A., Balluchi A., Prattichizzo D., Gorelli A., "Introducing the «Sphericle»: An experimental testbed for research and teaching in nonholonomy", Proc. IEEE Internat. Conf. on Robotics and Automation (New Mexico, 1997): vol. 3, pp. 2620-2625.

8. Michaud F., Caron S. "Roball, the rolling robot", Auton. Robots, 2002, vol. 12, pp. 211-222.

9. Alves J., Dias J. "Design and control of a spherical mobile robot”, J. Syst. Control Eng., 2003, vol. 217, pp. 457-467.

10. Halme A., Schonberg T., Wang Y. "Motion control of a spherical mobile robot", Proc. of the 4th IEEE Internat. Workshop on Advanced Motion Control (Mie, Japan, 1996): Vol. 1, pp. 259-264.

11. Kilin A. A., Pivovarova E. N., Ivanova T. B., "Spherical Robot of Combined Type: Dynamics and Control”, Regul. Chaotic Dyn., 2015, vol. 20, no. 6, pp. 716-728.

12. Svinin M., Bai Y., Yamamoto M. "Dynamic model and motion planning for a pendulumactuated spherical rolling robot", Proc. of the 2015 IEEE Internat. Conf. on Robotics and Automation (ICRA), pp.656-661.

13. Tomi J. Ylikorpi, Aarne J. Halme, Pekka J. Forsman, "Dynamic modeling and obstaclecrossing capability of flexible pendulum-driven ball-shaped robots", Robotics and Autonomous Systems, Volume 87, 2017, Pages 269-280. 
14. Ivanova T. B., Kilin A. A., Pivovarova E. N., "Controlled Motion of a Spherical Robot with Feedback. I", Journal of Dynamical and Control Systems, 2018, vol. 24, no. 3, pp. 497-510

15. Tafrishi S., Svinin S., Esmaeilzadeh E., Yamamoto M., "Design, Modeling, and Motion Analysis of a Novel Fluid Actuated Spherical Rolling Robot", J. Mechanisms Robotics. 2019, vol. 11, no. 4, pp. 041010.

16. Akella P., O'Reilly O.M., Sreenath K., "Controlling the Locomotion of Spherical Robots or Why BB-8Works", J. Mechanisms Robotics. 2019, vol. 11, no. 2, pp. 024501.

17. Borisov A.V., Kilin A.A., Mamaev I.S. "How to control Chaplygin's sphere using rotors", Regul. Chaotic Dyn., 2012, vol.17, nos.3-4, pp.258-272.

18. Borisov A.V., Kilin A.A., Mamaev I.S. "How to control the Chaplygin ball using rotors: 2", Regul.Chaotic Dyn., 2013, vol.18, nos.1-2, pp.144-158.

19. Morinaga A., Svinin M., Yamamoto M. "A motion planning strategy for a spherical rolling robot driven by two internal rotors”, IEEE Trans. on Robotics, 2014, vol.30, no.4, pp. 9931002.

20. Jia Q., Zheng Y., Sun H., Cao H., Li H. Motion control of a novel spherical robot equipped with a flywheel // ICIA'09: Internat. Conf. on Information and Automation (Zhuhai, Macau, 2009), pp. 893-898.

21. Schroll G.C. "Design of a spherical vehicle with flywheel momentum storage for high torque capabilities", Dissertation (bachelor of science), MIT, Cambridge, MA, 2008.

22. Joshi V. A., Banavar R. N. "Motion analysis of a spherical mobile robot", Robotica, 2009, vol. 27 , no. 3, pp. 343-353.

23. Sugiyama Y., Hirai S. "Crawling and jumping by a deformable robot", Int. J. Robot. Res., 2006, vol. 25, pp. 603-620.

24. "MorpHex, the incredible hexapod robot", https://youtu.be/yn3FWb-vQQ4

25. Mukherjee R., Minor M. A., Pukrushpan J. T. "Simple motion planning strategies for spherobot: a spherical mobile robot", Proc. of the 38th IEEE Conf. on Decision and Control (Phoenix, AZ, Dec 1999): Vol. 3, pp. 2132-2137.

26. Javadi A., Mojabi P. "Introducing glory: a novel strategy for an omnidirectional spherical rolling robot”, J. Dyn. Syst. Meas. Control Trans. ASME, 2004, vol. 126, no. 3, pp. 678683.

27. Karavaev Y. L., Kilin A. A., "Nonholonomic Dynamics and Control of a Spherical Robot with an Internal Omniwheel Platform: Theory and Experiments", Proceedings of the Steklov Institute of Mathematics, 2016, vol. 295, pp. 158-167

28. Chen W.-H., Chen Ch.-P., Yu W.-Sh., Lin Ch.-H., Lin P.-Ch. "Design and implementation of an omnidirectional spherical robot Omnicron", IEEE/ASME Internat. Conf. on Advanced Intelligent Mechatronics (Kachsiung, Taiwan, 2012), pp. 719-724.

29. Bhattacharya S., Agrawal S. "Spherical rolling robot: a design and motion planning studies", IEEE Trans. Robot. Autom., 2000, vol. 16, no. 6, pp. 835-839.

30. Roozegar M., Mahjoob M. J. "Modelling and control of a non-holonomic pendulum-driven spherical robot moving on an inclined plane: simulation and experimental results", IET Control Theory \& Applications, 2016, vol. 11, no. 4. pp. 541-549.

31. Borisov A. V., Kilin A. A., Karavaev Y. L., Klekovkin A. V., "Stabilization of the motion of a spherical robot using feedbacks", Applied Mathematical Modelling, 2019, vol. 69 , pp. 583-592

32. Borisov, A.V., Mamaev, I. S., and Karavaev, Yu. L., "On the loss of contact of the Euler disk. Nonlinear Dynam.”, 2015, vol. 79, no. 4, pp. 2287-2294.

33. Borisov, A. V. and Mamaev, I. S., "Strange Attractors in Rattleback Dynamics", PhysicsUspekhi, 2003, vol.46, no.4, pp. 393-403.

34. Ivanov, A. P., "On Detachment Conditions in the Problem on the Motion of a Rigid Body on a Rough Plane”, Regul. Chaotic Dyn., 2008, vol.13, no. 4, pp. 355-368. 